(RESEARCH ARTICLE)

\title{
Leptospirosis and scrub typhus co-infection in febrile patients
}

\author{
Sengupta Mallika *, Mukherjee Tamasi, Dasgupta Rajat and Banerjee Parthajit \\ Department of Microbiology, KPC Medical College, Kolkata, India.
}

Publication history: Received on 14 June 2020; revised on 20 June 2020; accepted on 23 June 2020

Article DOI: https://doi.org/10.30574/wjarr.2020.6.3.0202

\begin{abstract}
Leptospirosis has to be differentiated from other aetiologies of acute febrile illness like scrub typhus, malaria, enteric fever, dengue and spotted fever. Concurrent infection of leptospirosis can occur with typhoid fever, brucellosis and rickettsial infections. A study was done in a tertiary care centre in Eastern India to determine simultaneous presence of infection of leptospirosis and scrub typhus in sera of febrile patients. IgM antibodies to both leptospira and scrub typhus were detected by Enzyme linked immunosorbant assay (ELISA). During the study period of two years a total of 608 serum samples were received for serology for leptospirosis. Out of these samples 26 patients had IgM antibodies to leptospira, of which 11 (42.31\%) also had IgM antibodies to scrub typhus. As both leptospirosis and scrub typhus present with similar clinical features, co-infection of these two diseases may occur and it requires molecular methods for confirmation of these cases.
\end{abstract}

Keywords: Leptospirosis; Scrub typhus; Co-infection; ELISA

\section{Introduction}

Leptospirosis is a zoonotic disease caused mainly by pathogenic species under the genus Leptospira [1]. This infection is re-emerging in China, Japan, Australia, India and Europe. In India outbreaks have been reported from the Andaman Island, Tamil Nadu, Karnataka, Maharashtra, Andhra Pradesh and Orissa especially after heavy rains [2]. Most infections caused by leptospira are asymptomatic or subclinical infection. The common symptoms of leptospirosis are non-specific and include fever, headache, myalgia especially in the calf muscle, abdominal pain, conjunctival suffusion, and sometimes a skin rash [1].

Leptospirosis may present as an acute undifferentiated febrile illness. The different aetiologies of acute febrile illness are scrub typhus, malaria, enteric fever, dengue, leptospirosis and spotted fever [3]. Thus laboratory confirmation of disease is important as clinical management differ in these conditions.

Leptospira infection can occur in patients with other diseases. Concurrent infection of leptospirosis can occur with typhoid fever, brucellosis and rickettsial infection [4]. Simultaneous detection of antibodies to leptospira and scrub typhus has been reported in a few cases. This study was done to detect antibodies to scrub typhus in patients with acute fever having antibodies to leptospira.

\section{Material and methods}

A study was done for a period of two years (January 2018 to December 2019) in a tertiary care hospital in Eastern India to determine simultaneous infection of leptospirosis and scrub typhus. After obtaining ethical clearance from the institution and informed consent from the patients, sera of the adult patients having acute undifferentiated fever $\left(\geq 100^{\circ}\right.$ F), who were clinically suspected as leptospirosis were tested for leptospirosis serology. IgM antibodies to leptospirosis were detected by ELISA by PanBio kit (PanBio Ltd, Brisbane, Australia) according to the manufacturer's instructions

\footnotetext{
* Corresponding author: Sengupta Mallika, Email:mallikacyber@gmail.com
} 
and a value of 20 Panbio units was taken as positive [5]. These leptospira positive serum samples were further tested for co-infection with scrub typhus. IgM antibodies to scrub typhus were determined by ELISA InBios kit (InBios International Inc, Seattle, WA) according to the manufacturer's instructions and a cut-off of 0.5 of optical density (OD) was taken as positive. The clinical features, laboratory parameters and other data were entered in the excel spreadsheet (Microsoft Office, Redmond, Washington, USA) and analysis was done by using STATA version 20.

\section{Results}

During the study period of two years a total of 608 serum samples were received for serology for leptospirosis. Out of these samples 26 patients had IgM antibodies to leptospira as detected by ELISA. This showed that there was a serological prevalence of $4.28 \%$ among patients with acute febrile illness. These 26 serum samples were further tested for IgM antibodies to scrub typhus. Of these patients, $18(69.23 \%)$ were male and $8(30.77 \%)$ were female. There were $7(26.92 \%)$ patients of 18 - 30 years age, $15(57.69 \%)$ of $31-59$ years and $4(15.38 \%)$ of $\geq 60$ years of age.

Among these 26 patients 11 (42.31\%) also had IgM antibodies to scrub typhus detected by ELISA. The value of leptospira positive samples was between 20 to 54 Panbio units (mean $=25.4$, SD $=6.89$ ). The optical density of scrub typhus IgM ELISA for these samples was $0.026-3.314$ ( mean $=0.748$, SD =1.31).

Among the clinical features no significant correlation could be found between lone infection with leptospirosis and combined infection by leptospirosis and scrub typhus in terms of abdominal pain, vomiting, decreased urine output and acute respiratory distress syndrome (ARDS). Meningitis was seen in 2 patients and both of them had infection by both leptospira and Orientia tsutsugamushi. There were $8(72.72 \%)$ patients with co-infection of leptospirosis and scrub typhus and 7 (46.67\%) patients with only leptospirosis who had increased bilirubin level. Again, there were 6 (54.54\%) patients with co-infection of leptospirosis and scrub typhus and 3 (20\%) patients with only leptospirosis who had increased creatinine levels (Table 1). No significant correlation could be found between single infection or combined infection in case of raised bilirubin and creatinine $(p<0.05)$. Only myalgia was found to be significantly related to combined infection (Table 1).

Table 1 Shows the clinical parameters in included patients

\begin{tabular}{llll}
\hline & $\begin{array}{l}\text { Only leptospirosis } \\
(\mathbf{n = 1 5})\end{array}$ & $\begin{array}{l}\text { Leptospirosis + Scrub } \\
\text { typhus (n= 11) }\end{array}$ & p value \\
\hline Myalgia & 5 & 9 & 0.021 \\
Abdominal pain & 5 & 7 & 0.232 \\
Nausea and vomiting & 6 & 6 & 0.692 \\
Decreased urine output & 7 & 8 & 0.246 \\
ARDS & 4 & 3 & 1.000 \\
Bilirubin $\geq 1 \mathrm{mg} / \mathrm{dl}$ & 7 & 8 & 0.246 \\
Creatinine $\geq 1.4 \mathrm{mg} / \mathrm{dl}$ & 3 & 6 & 0.103 \\
\hline
\end{tabular}

\section{Discussion}

There are various tests for diagnosis of leptospirosis like loop mediated isothermal amplification assay, polymerase chain reaction (PCR) and enzyme linked immunosorbant assay [6]. The advantage of ELISA is that it can be performed easily with less infrastructure and technical expertise and is inexpensive compared to microscopic agglutination test (MAT). In addition, the ELISA can be automated, the result is objective, especially once a diagnostic cut-off has been decided upon, therefore having less interobserver / intraobserver variation. Since many other diseases have similar clinical manifestations the ELISA should be validated. The usage of well characterized archived specimens, consisting of those with disease and other clinically similar diseases allows rapid assessment of proof of concept and validation of the new assay [7]. This ELISA for leptospirosis has been validated in India and a diagnostic cut-off of 20 Panbio units has been determined [8]. IgM ELISA is the most widely used method for diagnosis of leptospirosis. Even Modified Faine's criteria uses IgM ELISA for diagnosis of leptospirosis [9]. 
In 2003, a case of concurrent infection of scrub typhus and leptospira was reported in a patient with acalculous cholecystitis [10]. In 2012, another case of co-infection with scrub typhus and leptospira was reported by Wei et al. However, both the cases responded well to management [11]. A similar case was reported from the Himalayan region in India in 2012 [12]. In this study we found co-infection of leptospira and scrub typhus in 11 (42.31\%) of patients by ELISA which is similar to the finding of Borkakoty et al who found scrub typhus IgM and leptospira IgM positive simultaneously in $25 \%$ cases of patients with pyrexia of unknown origin [13].

In 2013, a study by Sonthayanon et al, 82 cases with serological evidence of scrub typhus (by immunofluorescent assay or IFA) and leptospirosis (by MAT) were tested by duplex PCR targeting the 16S rRNA gene of both leptospira and Orientia tsutsugamushi to confirm or rule out co-infection. Both scrub typhus and leptospira specific nucleic acid was detected in 6\% (5/82), whereas specific DNA for only scrub typhus was detected in 5\% (4/82) and for only leptopsira in 46\% (38/82) [14]. This demonstrates that molecular evidence alone cannot be used to confirm or eliminate coinfection. This is especially true when samples are tested later in the course of illness as from second week onwards where serological assays are more likely to be positive. In this study, among the 11 samples with serological evidence of co-infection with scrub typhus and leptospirosis, around 75\% had fever of duration more than 7 days.

Elevated bilirubin or creatinine levels could not be significantly related to isolated infection by leptospirosis or dual infection. This was also shown in a study done Lee et al where fever, headache, jaundice and oliguria were not significantly related to leptospirosis versus dual infection of leptospirosis and scrub typhus [15].

\section{Conclusion}

Since both leptospirosis and scrub typhus present with similar clinical features, co-infection of these two diseases may occur and it requires different methods for confirmation of these cases. As, molecular confirmation of leptospirosis and scrub typhus was beyond the scope of this study and paired sera was not available and ELISA for detection of IgG antibodies could not be done, the issue of co-infection could not be fully resolved in these patients.

\section{Compliance with ethical standards}

\section{Disclosure of conflict of interest}

The authors declare that there is no conflict of interest.

\section{Statement of ethical approval}

The study was approved by the institutional ethical committee.

\section{Statement of informed consent}

Informed consent was obtained from all individual participants included in the study.

\section{References}

[1] Levett PN. Leptospirosis. (2001). Clin Microbiol Rev, 14(2), 296-326.

[2] Sambasiva RR, Naveen GPB and Agarwal SK. (2003). Leptospirosis in India and the rest of the world. Braz J Infect Dis off Publ Braz Soc Infect Dis, 7(3), 178-93.

[3] Chrispal A, Boorugu H, Gopinath KG, Chandy S, Prakash JAJ, Thomas EM, et al. (2010). Acute undifferentiated febrile illness in adult hospitalized patients: the disease spectrum and diagnostic predictors - an experience from a tertiary care hospital in South India. Trop Doct, 40(4), 230-4.

[4] Parker TM, Murray CK, Richards AL, Samir A, Ismail T, Fadeel MA, et al. Concurrent Infections in Acute Febrile Illness Patients in Egypt.

[5] Desakorn V, Wuthiekanun V, Thanachartwet V, Sahassananda D, Chierakul W, Apiwattanaporn A, et al. (2012). Accuracy of a Commercial IgM ELISA for the Diagnosis of Human Leptospirosis in Thailand. Am J Trop Med Hyg. $86(3), 524-7$. 
[6] Sengupta M, Prabhakar AKP, Satyendra S, Thambu D, Abraham OC, Balaji V, et al. (2017). Utility of Loop-mediated Isothermal Amplification Assay, Polymerase Chain Reaction, and ELISA for Diagnosis of Leptospirosis in South Indian Patients. J Glob Infect Dis, 9(1), 3-7.

[7] TDR Diagnostics Evaluation Expert Panel, Banoo S, Bell D, Bossuyt P, Herring A, Mabey D, et al. (2010). Evaluation of diagnostic tests for infectious diseases: general principles. Nat Rev Microbiol. 8(12 Suppl), S17-29.

[8] Sengupta M, Daniel D and Prakash JAJ. (2018). Determination of Cut-off for Leptospira Immunoglobulin M Enzyme-linked Immunosorbent Assay in South India, 6(8), 4.

[9] Dutta TK and Christopher M. (2005). Leptospirosis-an overview. Japi, 53, 545-551.

[10] Wang N-C, Ni Y-H, Peng M-Y and Chang F-Y. (2003). Acute acalculous cholecystitis and pancreatitis in a patient with concomitant leptospirosis and scrub typhus. J Microbiol Immunol Infect Wei Mian Yu Gan Ran Za Zhi, 36(4), 285-7.

[11] Wei Y-F, Chiu C-T, Lai Y-F, Lai C-H and Lin H-H. (2012). Successful treatment of septic shock and respiratory failure due to leptospirosis and scrub typhus coinfection with penicillin, levofloxacin, and activated protein $\mathrm{C}$. J Microbiol Immunol Infect Wei Mian Yu Gan Ran Za Zhi, 45(3), 251-4.

[12] Mahajan SK, Rolain J-M, Kashyap R, Gupta D, Thakur S, Sharma A, et al. (2009). Scrub typhus complicating pregnancy. J Assoc Physicians India, 57, 720-1.

[13] Borkakoty B, Jakharia A, Biswas D and Mahanta J. (2016). Co-infection of scrub typhus and leptospirosis in patients with pyrexia of unknown origin in Longding district of Arunachal Pradesh in 2013. Indian J Med Microbiol, 34(1), 88-91.

[14] Sonthayanon P, Chierakul W, Wuthiekanun V, Limmathurotsakul D, Amornchai P, Smythe LD, et al. (2013). Molecular confirmation of co-infection by pathogenic Leptospira spp. and Orientia tsutsugamushi in patients with acute febrile illness in Thailand. Am J Trop Med Hyg, 89(4), 797-9.

[15] Lee C-H and Liu J-W. (2007). Coinfection with leptospirosis and scrub typhus in Taiwanese patients. Am J Trop Med Hyg, 77(3), 525-527.

\section{How to cite this article}

Sengupta M, Mukherjee T, Dasgupta R and Banerjee P. (2020). Leptospirosis and scrub typhus co - infection in febrile patients. World Journal of Advanced Research and Reviews, 6(3), 233-236. 